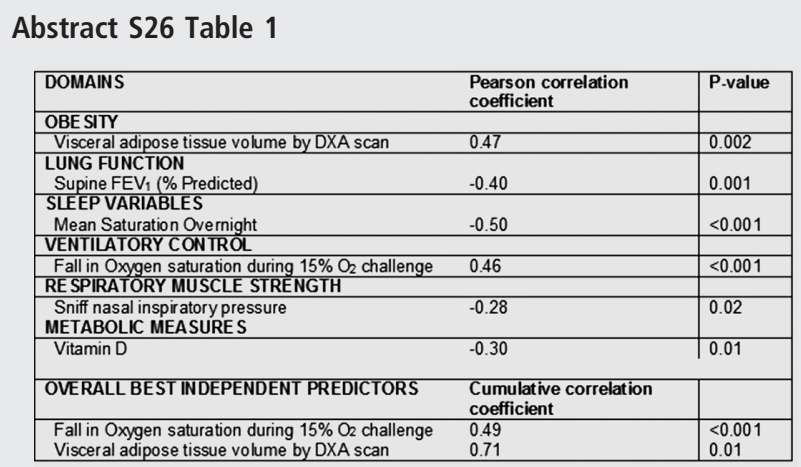

The table shows the correlation coefficient for the statistically strongest predictors of a raised $B E$ in each domain by multiple linear regression. The bottom of the table shows the overall outcome of the multiple linear regression, when each of the strongest independent predictors were matched against each other.

individuals, the presence of a raised plasma standard bicarbonate (or base excess, $\mathrm{BE}$ - a biomarker of whole body acid-base balance, including overnight $\mathrm{PaCO}_{2}$ levels), without necessarily a raised daytime $\mathrm{PaCO}_{2}$, has been shown by us to be an intermediary stage towards overt obesity-hypoventilation syndrome. Thus we have looked for biologically plausible predictors of a raised base excess in obesity, whether or not there was also a raised $\mathrm{PaCO}_{2}$ awake.

Methods 78 obese subjects (BMI $>30$, mean 47 (SD 10, range 32 to 74$) \mathrm{kg} / \mathrm{m}^{2}$ ) were identified from a variety of sources, regardless of their $\mathrm{PaCO} 2$ and acid/base status (mean levels 5.6 (SD 0.8, range 4.2 to 9.6 ) $\mathrm{kPa}$; and 2.1 (SD 2.4, range -3.5 to 10) $\mathrm{mmol} / \mathrm{l}$ respectively) and a large number of their characteristics measured. Biological plausible domains were constructed that were thought potentially to contribute to any ventilatory failure. First, the best independent predictor of the $\mathrm{BE}$ within each domain was found, second, the best overall independent predictors were found. The domains were as follows:

- Obesity and its distribution (BMI, simple surface measures, DXA [a radiographic derivative])

- Lung function (sitting/lying spirometry and forced oscillometry)

- Sleep variables (AHI, ODI, mean overnight $\mathrm{SaO} 2$, time below 90\% SaO2)

- Ventilatory control (2 point responses to $15 \%$ O2 and 5\% $\mathrm{CO} 2)$

- Respiratory muscle strength (mouth pressures, sniff pressures)

- Metabolic measures (e.g. leptin, adipokines, vitamin D)

Conclusions There are a number of strong predictors for the presence of a raised base excess in obesity. Significant predictors were found in each of the biological domains we studied, suggesting that the cause of ventilatory failure in obesity is likely to be multifactorial. However, reduced hypoxic poikilocapnic ventilatory drive and the presence of intra-abdominal obesity seem to be the most powerful predictors of a raised base excess in obesity.

\section{S27 VENOUS BICARBONATE AS A CLINICAL TOOL FOR IDENTIFYING OBESITY HYPOVENTILATION SYNDROME IN THE SLEEP CLINIC}

B Prudon, SD West. Newcastle Regional Sleep Service, Newcastle Upon Tyne, UK

10.1136/thoraxjnl-2014-206260.33

Introduction Obesity Hypoventilation Syndrome (OHS) is defined as sleep disordered breathing, obesity, and daytime hypercapnia, without another cause of ventilatory impairment.
Literature suggests $10-25 \%$ of patients assessed for Obstructive Sleep Apnoea (OSA) have OHS, with significantly increased morbidity and mortality. Early identification may be beneficial. Studies suggest venous bicarbonate $\left(\mathrm{vHCO}_{3}{ }^{-}\right) \geq 27 \mathrm{mmol} / \mathrm{l}$ can be used to screen for OHS. We assessed the impact of incorporating this measurement into patient assessments.

Methods Obese out-patients referred for possible OSA had $\mathrm{vHCO}_{3}{ }^{-}$measured. Patients with a $\mathrm{vHCO}_{3}{ }^{-} \geq 27 \mathrm{mmol} / \mathrm{l}$ underwent arterial blood gas (ABG) analysis. Those with $\mathrm{pCO}_{2}>6.2$ $\mathrm{kPa}$ underwent further assessments to identify the cause of ventilatory impairment. None had been referred specifically for investigation of OHS. Patients had domiciliary or in-patient sleep studies as per standard practice.

Results There were 288 patients included: 65\% males, mean (SD) age 50 years (range 21-79 years), BMI $39.2 \mathrm{~kg} / \mathrm{m}^{2}$ (7.8), Epworth Sleepiness Scale 13 (6), daytime $\mathrm{SpO}_{2}$ on air 97\% (2.1). Sleep study results showed the Apnoea-Hypopnea Index (AHI) to be $\geq 5$ in $88 \%$, and $\geq 30$ in $49 \%$. Mean $\mathrm{vHCO}_{3}{ }^{-}$was $26.2 \mathrm{mmol} / \mathrm{l}(2.7) . \mathrm{vHCO}_{3}{ }^{-}$correlated significantly $(\mathrm{r}=0.3-0.4$, $\mathrm{p}<0.005)$ with daytime $\mathrm{SpO}_{2}$, mean overnight $\mathrm{SpO}_{2}$, time spent $<80 \%$ and $<90 \%$, but not AHI or ODI.

$\mathrm{vHCO}_{3}{ }^{-}$was $\geq 27 \mathrm{mmol} / \mathrm{l}$ in 123 (43\%), of whom 80 had an ABG measurement; mean $\mathrm{pCO}_{2} 5.4 \mathrm{kPa}(0.8)$, ten patients $>6.2$ $\mathrm{kPa}$. Ventilatory impairment was due to $\mathrm{OHS}$ in four $(5 \%$ of ABG cohort); there was additional lung or chest wall disease in the other six. Overall, 25 patients had a base excess $\geq 3$. The $\mathrm{vHCO}_{3}{ }^{-}$range was $28-36 \mathrm{mmol} / \mathrm{l}$ in patients with $\mathrm{OHS}$, with a BMI range of $38-53 \mathrm{~kg} / \mathrm{m}^{2}$.

Three additional outpatients with $\mathrm{BMI}>50 \mathrm{~kg} / \mathrm{m}^{2}$ were diagnosed with $\mathrm{OHS}$ on $\mathrm{ABG}$ without $\mathrm{vHCO}_{3}{ }^{-}$measurement. In all seven OHS patients, CPAP was initiated. One was non-compliant, four improved and two required home non-invasive ventilation due to non-improvement in ABG.

Conclusions In this large cohort of patients assessed for OSA, $43 \%$ had a $\mathrm{vHCO}_{3}^{-} \geq 27 \mathrm{mmol} / \mathrm{l}$ indicating possible OHS, but only $5 \%$ were actually diagnosed with OHS. In isolation this strategy to identify OHS seems inefficient. An increased $\mathrm{vHCO}_{3}{ }^{-}$ in combination with sleep study data may be superior.

\section{S28 LIRAGLUTIDE 3.0 MG REDUCES SEVERITY OF OBSTRUCTIVE SLEEP APNOEA AND BODY WEIGHT IN OBESE INDIVIDUALS WITH MODERATE OR SEVERE DISEASE: SCALE SLEEP APNOEA TRIAL}

${ }^{1} \mathrm{~A}$ Collier, ${ }^{2} \mathrm{~A}$ Blackman, ${ }^{3} \mathrm{G}$ Foster, ${ }^{4} \mathrm{G}$ Zammit, ${ }^{5} \mathrm{R}$ Rosenberg, ${ }^{6} \mathrm{~T}$ Wadden, ${ }^{7} \mathrm{~L}$ Aronne, ${ }^{8} \mathrm{~B}$ Claudius, ${ }^{8} \mathrm{~T}$ Jensen, ${ }^{9} \mathrm{E}$ Mignot. ${ }^{7}$ Ayr Hospital, Ayr, UK; ${ }^{2}$ Toronto Sleep Institute, MedSleep, Toronto, Canada; ${ }^{3}$ Temple University, Philadelphia, USA; ${ }^{4}$ Clinilabs Sleep Disorders Institute, New York, USA; ${ }^{5}$ NeuroTrials Research, Atlanta, USA; ${ }^{6}$ University of Pennsylvania, Philadelphia, USA; ${ }^{7}$ Weill Cornell Medical College, New York, USA; ${ }^{8}$ Novo Nordisk A/S, Soborg, Denmark; ${ }^{9}$ Stanford University, Palo Alto, USA

\subsection{6/thoraxjnl-2014-206260.34}

Aims/objectives This randomised, double-blind, parallel-group trial compared the effects of liraglutide $3.0 \mathrm{mg}$ to placebo, both as adjunct to diet and exercise, on obstructive sleep apnoea (OSA) severity and body weight.

Content Obese individuals $(\mathrm{n}=359)$ without diabetes who had moderate or severe OSA and were unwilling/unable to use continuous positive airway pressure therapy were randomised 1:1 to liraglutide $3.0 \mathrm{mg}$ or placebo for 32 weeks (baseline characteristics: 48.5 years, males $71.9 \%$, apnoea-hypopnoea index [AHI] 49.2 events/h, body weight $117.6 \mathrm{~kg}$, BMI $\left.39.1 \mathrm{~kg} / \mathrm{m}^{2}, \mathrm{HbA}_{1 \mathrm{c}} 5.7 \%\right)$. Outcomes At end-of-trial, the reduction in AHI was significantly greater with liraglutide $3.0 \mathrm{mg}$ than placebo (Table). Liraglutide 